\title{
Relationship between osteoporosis and expression of Bcl-2 and CXCL12
}

\author{
TONGTAO PANG, MINGZHI GONG, JIANGTAO HAN and DAN LIU
}

\begin{abstract}
Department of Trauma and Orthopedics, The Second Hospital of Shandong University, Jinan, Shandong 250033, P.R. China
\end{abstract}
Received July 19, 2017; Accepted November 2, 2017

DOI: 10.3892/etm.2017.5513

\begin{abstract}
The changes of expression of anti-apoptotic factor B-cell lymphoma 2 (Bcl-2) and chemokine C-X-C motif ligand 12 (CXCL12) in the pathological process of osteoporosis (OP) were investigated, to provide new ideas for the diagnosis and treatment of OP. A total of 60 postmenopausal women who needed to undergo hip replacement surgery were enrolled and divided into osteoporosis group (OP, $n=32)$ and control group ( $\mathrm{CK}, \mathrm{n}=28)$ according to the results of dual-energy $\mathrm{X}$-ray bone density measure; after operation, cancellousbone from the femoral head or femoral neck was removed, and osteoblasts and osteoclasts were isolated and cultured in vitro. The proliferation and apoptosis in the two groups of osteoblasts and osteoclasts were detected by 3-(4,5-dimethylthiazol2-yl)-2,5-diphenyltetrazolium bromide (MTT) colorimetry and Annexin V/PI double staining method, respectively. The expression levels of Bcl-2 and CXCL12 mRNA and protein in the two groups of osteoblasts and osteoclasts were determined by reverse transcription quantitative polymerase chain reaction (RT-qPCR) and western blot analysis. The analysis of cell proliferation and apoptosis showed that compared with the CK group, osteoblast proliferation was significantly inhibited and apoptosis rate was distinctly increased in the OP group, compared with the CK group, osteoclast proliferation was distinctly enhanced and apoptosis rate was remarkably reduced in the OP group. The results of RT-qPCR and western blot analysis displayed that Bcl-2 and CXCL12 mRNA and protein levels in osteoblasts of the OP group were significantly lower than those of the $\mathrm{CK}$ group, while mRNA and protein levels of Bcl-2 and CXCL12 in osteoclast of the OP group were distinctly increased compared to those in the CK group. The incidence of OP is closely associated with the bone balance maintained by osteoblasts and osteoclasts, and this mechanism may be achieved by inhibiting osteoblast proliferation
\end{abstract}

Correspondence to: Dr Mingzhi Gong, Department of Trauma and Orthopedics, The Second Hospital of Shandong University, 247 Beiyuan Street, Jinan, Shandong 250033, P.R. China

E-mail: mingzhigong@126.com

Key words: osteoporosis, B-cell lymphoma 2, chemokine C-X-C motif ligand 12 , gene expression and osteoclast apoptosis via regulating Bcl-2 and CXCL12 gene expression changes.

\section{Introduction}

Osteoporosis (OP) refers to the reduction of whole body bone mass and bone density as well as the degeneration of bone microstructure, leading to lower bone strength and increased bone fragility, which is accompanied by pathological fractures (1). OP is becoming more common with the increasing of age; therein, $\sim 45 \%$ of people over 50 years old suffer from $\mathrm{OP}$, which is more common in females than in males, with the rate as high as $9-38 \%$. The pathogenesis of OP is very complex, involving bone remodeling dysfunction, suppressive immune modulation disorders, inhibition of bone differentiation and vitamin D deficiency. In the physiological process of bone remodeling, the balance between bone absorption and bone formation is maintained by osteoblast and osteoclast; osteoclast can absorb and degrade bone matrix at multiple sites, while osteoblast can differentiate into new bone tissue in the bone resorption zone. When bone absorption is greater than bone formation, the bone balance is broken, that is, OP occurs (2).

The pathological process of OP is affected by multiple factors, including calcium ion, cytokines, estrogen, sunniness and diet. Among them, estrogen is extensively investigated, which is the decisive factor for the occurrence of OP after menopause. Estrogen secretion is decreased in postmenopausal women, leading to the reduction in combination with estrogen receptors on the surface of osteoblast and promoting cytokine release, thereby abnormally activating nuclear factor $-\kappa \mathrm{B}(\mathrm{NF}-\kappa \mathrm{B})$, affecting expression of downstream target genes (such as apoptosis factor family, chemokines and tumor necrosis factors), inhibiting osteoblast proliferation and differentiation, thus resulting in inducing OP (3). Studies have indicated that estrogen inhibits the secretion of cytokines in hematopoietic stem cells and osteoblasts, such as interleukin-1 (IL-1), tumor necrosis factor (TNF) and granulocyte-macrophage colony-stimulating factor (GM-CSF), which can promote osteoclast proliferation and differentiation and inhibit its apoptosis (4). Additionally, the activation of osteoclast is regulated by multiple signaling molecules, in which receptor activator of nuclear factor- $\kappa \mathrm{B}$ ligand (RANKL) is widely explored. It is produced by osteoblast and stimulates receptor activator of nuclear factor- $\kappa \mathrm{B}$ (RANK) to inhibit the 
$\mathrm{NF}-\kappa \mathrm{B}$ pathway and bone absorption ability of osteoclast (5). Chemokine C-X-C motif ligand 12 (CXCL12), namely stromal cell-derived factor-1 (SDF-1), is a downstream-related target gene of the $\mathrm{NF}-\kappa \mathrm{B}$ pathway. It is expressed in the brain, thymus, bone marrow and other tissues, and has strong chemotaxis to lymphocytes and mesenchymal stem cells. The study reveals that CXCL12 is highly expressed in inflammatory bone lesions and inhibits osteoclasts (6).

This study investigated the occurrence of $\mathrm{OP}$ and changes of expressions of anti-apoptotic factor B-cell lymphoma 2 (Bcl-2) and CXCL12 of downstream target genes of the $\mathrm{NF}-\kappa \mathrm{B}$ pathway in its pathological process, to provide new theoretical knowledge for the mechanism of bone balance maintained by osteoblast and osteoclast and ideas for the diagnosis and treatment of OP.

\section{Materials and methods}

\section{Materials}

General data. A total of 60 postmenopausal women admitted to the Department of Trauma and Orthopedics in Shandong Hospital, who needed to undergo hip replacement surgery from March 2015 to July 2016, were enrolled. The average age was $62 \pm 7.83$ years. Patients were divided into osteoporosis group (OP, $n=32$, average age $64 \pm 6.78$ years) and control group (CK, $n=28$, average age $61 \pm 8.53$ years) according to the results of dual-energy X-ray bone density measure. Patients with disagreement with the study, secondary OP or medication affecting bone metabolism in recent months were excluded. All patients and their family members were notified and signed the informed consent that met the ethical criteria. There was no significant difference in the age between the groups $(\mathrm{P}>0.05)$. The study was approved by the Ethics Committee of the Second Hospital of Shandong Universityand informed consents were signed by the patients and/or guardians.

Main reagents. Dulbecco's modified Eagle's medium (DMEM), fetal calf serum (Gibco; Thermo Fisher Scientific, Inc., Waltham, MA, USA); 1,25-(OH)2D3 (Sigma, St. Louis, MO, USA); 3-(4,5-dimethylthiazol-2-yl)-2,5-diphenyltetrazolium bromide (MTT) Cell Proliferation Assay kit (Trevigen, New York, NY, USA); Annexin V-FITC/PI Apoptosis Detection kit (eBioscience, Inc., San Diego, CA, USA); TRIzol Reagent, Prime Script ${ }^{\circledR}$ RT reagent kit with gDNA Eraser, SYBR $^{\circledR}$ Premix Ex Taq ${ }^{\mathrm{TM}}$ II (Takara Biotechnology Co., Ltd., Dalian, China); Cell Lysis Solution, polyvinylidene difluoride (PVDF) membrane (Roche, Basel, Switzerland); rabbit anti-human monoclonal antibodies of anti- $\beta$-actin, Bcl-2 and CXCL12 (dilution, 1:1,000; cat. nos. 8457, 4223 and 3530), horseradish peroxidase (HRP) goat anti-rabbit secondary polyclonal antibody (dilution, 1:2,000; cat. no. 7074) (CST, Boston, MA, USA); modified bicinchoninic acid (BCA) Protein Quantification kit, primers by Sangon Biotech (Shanghai) Co., Ltd., China.

\section{Methods}

Isolation and culture of osteoblast and osteoclast. Referring to methods reported by Robey and Termine (7), osteoblasts were isolated and cultured by modified enzyme digestion, followed by staining and cell morphology identification using modified Gomorialkaline phosphatase calcium-cobalt method. Refer-
Table I. Primers and sequences of RT-qPCR.

\begin{tabular}{ll}
\hline Gene & Primer sequence $\left(5^{\prime}-3^{\prime}\right)$ \\
\hline Bcl-2-F & TGAACCGGCATCTGCACAC \\
Bcl-2-R & CGTCTTCAGAGACAGCCAGGAG \\
CXCL12-F & ATTCTCAACACTCCAAACTGTGC \\
CXCL12-R & ACTTTAGCTTCGGGTCAATGC \\
ACTB-F & CAGGGCGTGATGGTGGGCA \\
ACTB-F & CAAACATCATCTGGGTCATCTTC
\end{tabular}

RT-qPCR, reverse transcription quantitative polymerase chain reaction.

ring to methods reported by MacDonald et al (8), osteoclast was isolated and cultured; by adopting human bone marrow as resource, bone marrow mononuclear cells were extracted and induced to differentiate into osteoclast-like cells in the presence of 1,25- $(\mathrm{OH})_{2} \mathrm{D}_{3}$, and the morphology of mature osteoclasts was observed and identified by tartrate-resistant acid phosphatase staining method.

Analysis of cell proliferation. The subcultured osteoblasts and osteoclasts in each group were treated by survival cell count and analysis according to the instructions of kit at intervals of $12 \mathrm{~h}$. The absorbance value of cells in each group was detected using enzyme analyzer (Tecan Group Ltd., Männedorf, Switzerland) at wavelength of $490 \mathrm{~nm}$. The data were expressed as an average percentage of living cells compared with that in the control group (\% of control).

Analysis of apoptosis. Apoptosis analysis was performed $48 \mathrm{~h}$ after subculture in accordance with the kit instructions. Annexin V-FITC/PI double staining was used for osteoblasts and osteoclasts. Apoptosis rate in early and late stages was analyzed using BD FACS Calibur flow cytometer (BD Biosciences, New York, NY, USA).

Real-time quantitative polymerase chain reaction (RT-qPCR) analysis. After subculture for 10 days, the total RNA in osteoblasts and osteoclasts was extracted and identified according to the instructions of the kits. cDNA was synthesized via reverse transcription using $200 \mathrm{ng}$ total RNA, and real-time fluorescent quantitative PCR system was prepared. cDNA was detected by CFX-96 Real-Time PCR Detection system (Bio-Rad, New York, NY, USA). The collected $\mathrm{Ct}$ value was processed into a relative expression form by comparing cycle threshold method with CK group as the control and $\beta$-actin as the internal reference; primer sequences are shown in Table I.

Western blot analysis. Osteoblast and osteoclast with 10 days subculture were lysed in cell lysate, and centrifuged at $4^{\circ} \mathrm{C}(2,600 \mathrm{x} \mathrm{g}$ for $5 \mathrm{~min})$. The supernatant was extracted, and protein concentration was determined using modified BCA Kit. A $50 \mu$ g protein sample was separated in $10 \%$ sodium dodecyl sulphate-polyacrylamide gel electrophoresis (SDS-PAGE), followed by electrical transfer printing on PVDF membrane. Subsequently, the sample was added with sealing fluid and sealed at room temperature for $2 \mathrm{~h}$, followed by incubation with rabbit anti- $\beta$-actin, Bcl- 2 and CXCL12 primary antibodies $(1: 1,000)$ at $4^{\circ} \mathrm{C}$. The next day, the sample was rinsed 
A

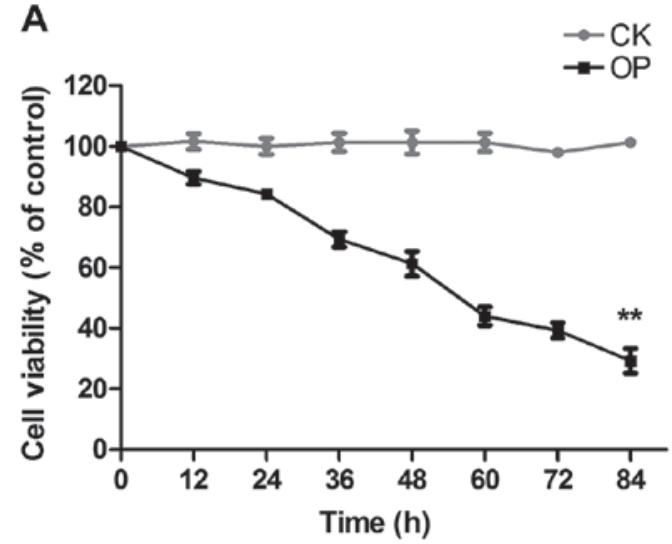

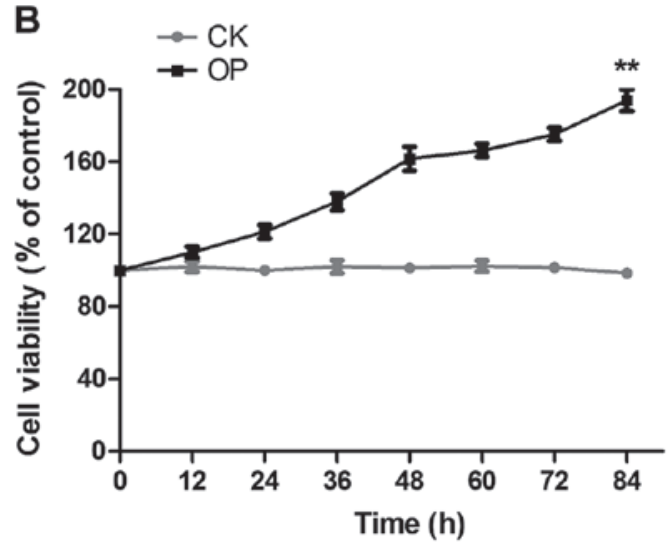

Figure 1. Osteoblast and osteoclast proliferation. (A) Osteoblast proliferation; (B) Osteoclast proliferation. The result of MTT colorimetry indicates that compared with the CK group, osteoblast proliferation is significantly inhibited in the OP group, while osteoclast proliferation is distinctly enhanced ${ }^{* *} \mathrm{P}<0.01$ vs. CK. CK group, control group; OP, osteoporosis.
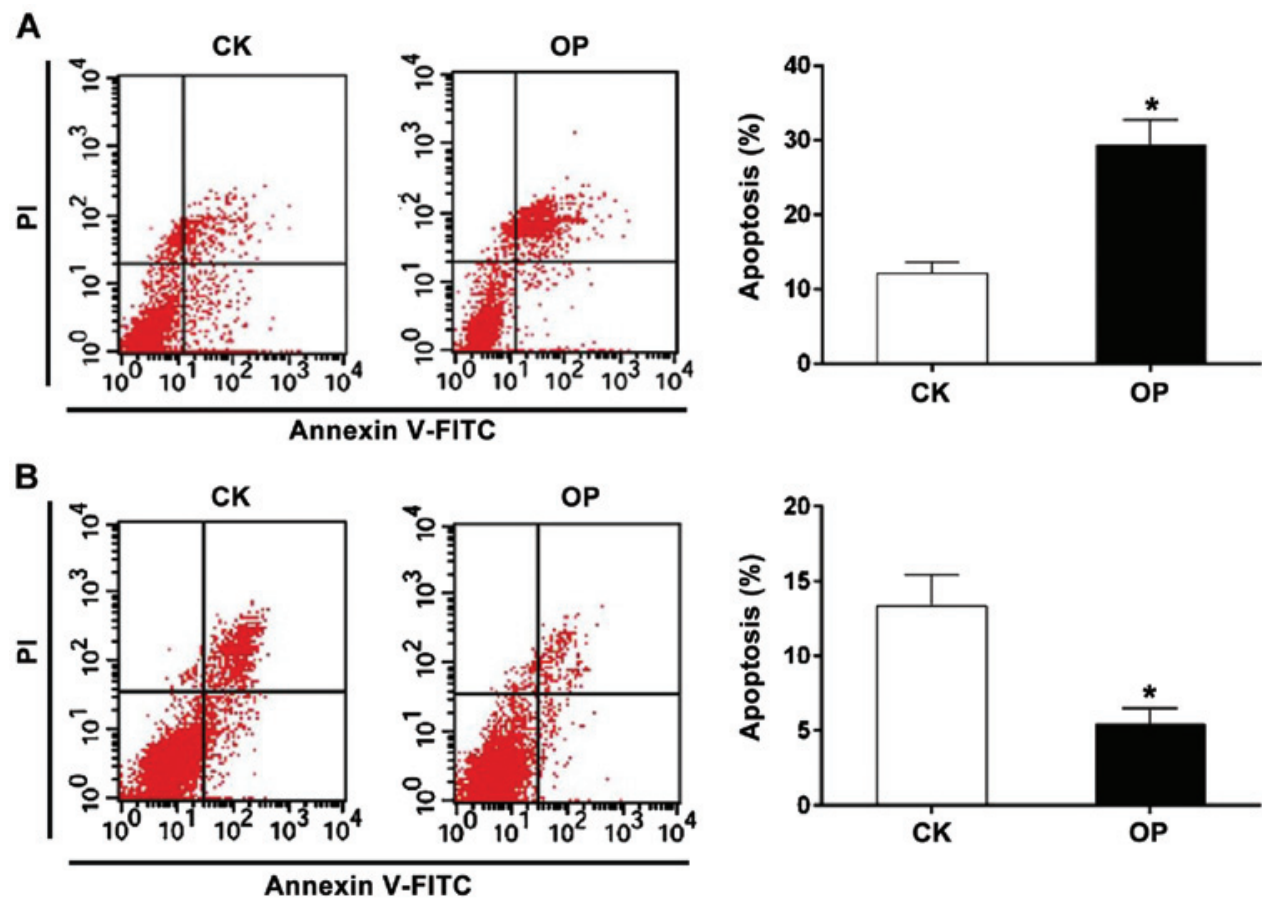

Figure 2. Osteoblast and osteoclast apoptosis. (A) Analysis of osteoblast apoptosis; (B) Analysis of osteoclast apoptosis. The result of Annexin V-FITC/PI double staining method reveals that the apoptosis rate of osteoblasts is significantly higher in the OP group than that in the CK group, while the apoptosis rate of osteoclasts is distinctly lower in the CK group. "P<0.05 vs. CK. OP, osteoporosis; CK group, control group.

by $0.05 \%$ Tris-buffered saline Tween (TBST) three times, followed by incubation with shock using HRP goat anti-rabbit secondary antibody $(1: 2,000)$ at room temperature for $2 \mathrm{~h}$. The bands were developed adopting electrogenerated chemiluminescence (ECL) detection system (Thermo Fisher Scientific, Inc.), and the gray value of band was calculated by Image $\mathbf{J}$ software (National Institutes of Health, Bethesda, MD, USA). The ratio of gray value of target protein to $\beta$-actin was set as the protein level.

Statistical analysis. SPSS 17.0 software (SPSS, Chicago, IL, USA) was adopted for statistical analysis. Data were expressed as mean \pm standard deviation (mean $\pm \mathrm{SD}$ ). Single-factor analysis of variance and double-tailed t-test were used for intergroup comparison. $\mathrm{P}<0.05$ suggested that the difference was statistically significant.

\section{Results}

Analysis of osteoblast and osteoclast proliferation. The proliferation of osteoblasts and osteoclasts in the two groups was detected by MTT colorimetry, as shown in Fig. 1: Fig. 1A displays osteoblast proliferation; compared with the CK group, osteoblast proliferation was significantly inhibited with the prolongation of culture time in the OP group; Fig. 1B represents osteoclast proliferation; compared with the CK group, osteoclast proliferation was distinctly enhanced in the OP group.

Analysis of osteoblast and osteoclast apoptosis. Apoptosis in the two groups was determined by Annexin V/PI, as shown in Fig. 2: 2A represents osteoblast apoptosis; compared with the CK group, the apoptosis rate in the early and late stage 
A

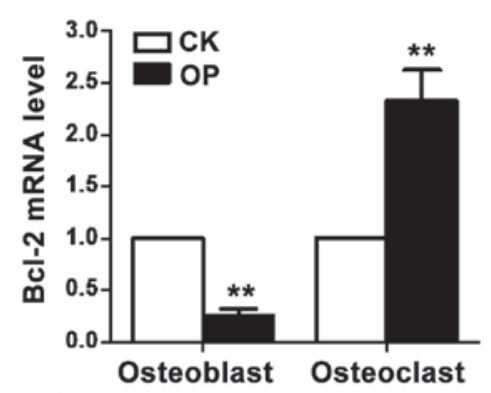

C

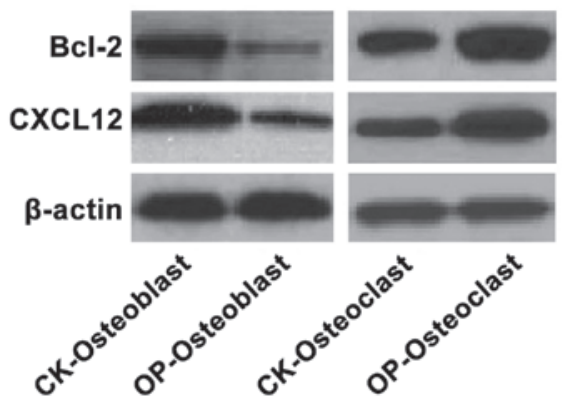

B

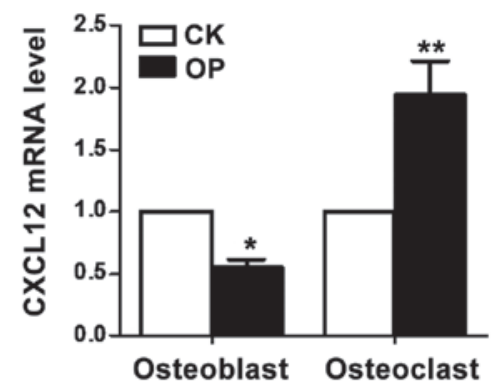

D $\square$ CK-Osteoblast

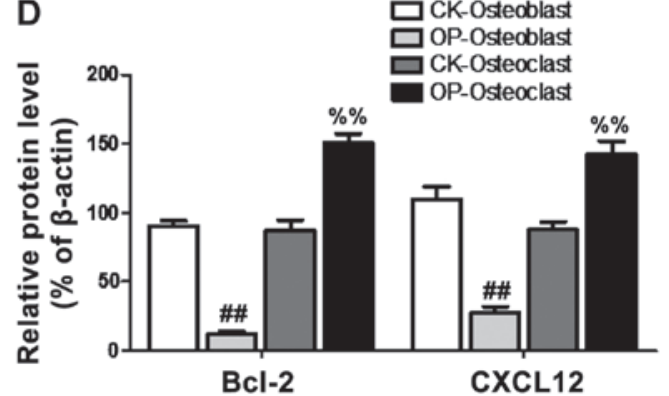

Figure 3. mRNA and protein levels of Bcl-2 and CXCL12 in osteoblast and osteoclast. (A) Bcl-2 mRNA level; (B) CXCL12 mRNA level; (C) protein expression of Bcl-2 and CXCL12; (D) relative levels of Bcl-2 and CXCL12 proteins. The results of RT-qPCR and western blot analysis indicate that mRNA and protein levels of Bcl-2 and CXCL12 in osteoblast of the OP group were significantly lower than those of the CK group, while the mRNA and protein levels of Bcl-2 and CXCL12 in osteoclast of the OP group were evidently higher than those of the CK group. ${ }^{*} \mathrm{P}<0.05$ vs. CK; ${ }^{* *} \mathrm{P}<0.01$ vs. CK; ${ }^{\# \#} \mathrm{P}<0.01$ vs. CK-Osteoblast; ${ }^{\%} \mathrm{P}<0.01$ vs. CK-Osteoclast. Bcl-2, B-cell lymphoma 2; CXCL12, chemokine C-X-C motif ligand 12; RT-qPCR analysis, real-time quantitative polymerase chain reaction analysis; OP, osteoporosis; CK group, control group.

was remarkably increased in the OP group; Fig. 2B indicates osteoclast apoptosis; compared with the CK group, the apoptosis rate in the early and late stages was obviously reduced in the OP group.

Changes of Bcl-2, CXCL12 mRNA and protein levels in osteoblast and osteoclast. Bcl-2 and CXCL12 mRNA and protein levels in osteoblasts and osteoclasts were quantitatively analyzed using RT-qPCR and western blot analysis, as shown in Fig. 3: Fig. 3A and B respectively represent mRNA levels of $\mathrm{Bcl}-2$ and CXCL12 in the two groups, indicating that mRNA levels of Bcl-2 and CXCL12 in osteoblasts of the OP group were significantly lower than those of the $\mathrm{CK}$ group, while mRNA levels of Bcl-2 and CXCL12 in osteoclasts of the OP group were distinctly increased; Fig. $3 \mathrm{C}$ and $\mathrm{D}$ reveals expression levels of Bcl-2 and CXCL12 proteins in the two groups of cells, suggesting that protein levels of Bcl-2 and CXCL12 in osteoblast of the OP group were significantly lower than those of the CK group, while protein levels of Bcl-2 and CXCL12 in osteoclasts of the OP group were distinctly increased.

\section{Discussion}

$\mathrm{OP}$ is a systemic metabolic bone disease due to the reduction of bone mass, loss of bone matrix, resulting in decreased bone density and destruction of bone tissue microstructure. According to statistics, 22 million women and 5.5 million men in the European Union suffer from OP, and whites and Asians are more likely to be affected (9). Moreover, the incidence of fractures caused by OP has exceeded myocardial infarction, stroke and other diseases, greatly endangering people's health.

The pathogenesis of OP is regulated by multiple factors and genes. Among them, the disorder in bone balance maintained by osteoclasts and osteoblasts is the direct factor of OP pathogenesis. The study indicates that bone matrix remodeling is constant in normal bone, which is maintained by bone metabolic units (BMUs) consisting of osteoblasts and osteoclasts (10). However, in the model of OP, the rate of bone matrix degradation by osteoclast is faster than that of bone rebuilt by osteoblasts, thereby accelerating the loss of bone mass and reduction of bone density. In the process of bone remodeling, cytokines secreted by BMUs are significant; therein, osteoprotegerin/osteoclastogenesis inhibitory factor (OPG/OCIF) secreted by osteoclasts inhibits the osteoblast transmission pathway and osteoclast formation (11). Transforming growth factor- $\beta$ (TGF- $\beta$ ) can promote the proliferation of progenitor cells of bone marrow osteoblast, thus increasing the number of osteoblasts; insulin-like growth factor-1 (IGF-1) can stimulate osteoblast proliferation and differentiation, and increase osteocalcin production.

Additionally, hormones play an important role in bone remodeling and bone absorption. The study shows that estrogen deficiency can increase bone absorption and reduce new bone deposition (12). The combination of estrogen and estrogen receptor (ER) exerts a significant effect in bone turnover. The study displays that the combination of estrogen and ER can stimulate osteoblast differentiation and increase bone matrix deposition (13). ER $\alpha$ is highly expressed in osteoclast 
precursors, but not expressed in mature osteoclasts (14). In addition, estrogen inhibits bone absorption by modulating the expression of c-Jun and phosphorylation of its N-terminal kinase (15). Furthermore, parathyroid hormone (PTH) and calcitonin can play important roles in bone turnover and bone deposition by regulating bone calcium metabolism (16). In recent years, it was revealed that genetic polymorphism is also related to $\mathrm{OP}$, which can determine the genetic predisposition of OP and affect the treatment of OP with estrogen (17).

The occurrence of OP can regulate the proliferation and apoptosis of osteoblast and osteoclast through various signaling pathways and their downstream target genes, resulting in reduction of bone mass and inducing fracture. Therein, the NF- $\mathrm{B}$ signaling pathway is closely associated with the pathological process of OP. The study indicates that osteoclast formation is inhibited in p50/p52 knockout mice (18). Multiple cytokines secreted by osteoblasts can promote the activation of $\mathrm{NF}-\kappa \mathrm{B}$ in the cells (19). Additionally, osteoclast differentiation is inhibited in $\mathrm{NF}-\kappa \mathrm{B} 1$ and $\mathrm{NF}-\kappa \mathrm{B} 2$ double knockout mouse models (20). The downstream target genes of the $N F-\kappa B$ pathway, such as apoptosis factor family and chemokines are of great significance in cell proliferation, differentiation, apoptosis, tumor formation and disease metabolism.

In this study, it is found that in postmenopausal OP patients, the proliferation of osteoblast was inhibited, the rate of apoptosis was increased significantly, while osteoclast proliferation was distinctly enhanced, and apoptosis was inhibited, indicating that in the pathological process of OP after menopause, osteoclast proliferation is greater than osteoblast proliferation and apoptosis of osteoblast is greater than apoptosis of osteoclast, breaking the status of bone balance maintained by these two factors, making bone degradation more than bone formation, thus leading to the occurrence and development of OP. Furthermore, the mRNA and protein expression of Bcl-2 and CXCL12 in osteoblasts of postmenopausal OP patients were decreased significantly, while the mRNA and protein levels of Bcl-2 and CXCL12 in osteoclasts were distinctly increased, suggesting that the pathological process of OP is closely related to the NF- $\kappa \mathrm{B}$ signaling pathway in postmenopausal women. The downstream target genes regulate the proliferation and apoptosis of osteoblasts and osteoclasts, destroy the balance of bone, make the process of bone remodeling disorder and accelerate the pathological process of OP. In summary, the incidence of OP is closely associated with the bone balance maintained by osteoblasts and osteoclasts, and this mechanism may be achieved by inhibiting osteoblast proliferation and osteoclast apoptosis via regulating Bcl-2 and CXCL12 gene expression changes. Moreover, the $\mathrm{NF}-\kappa \mathrm{B}$ pathway and its downstream target genes may provide new perspectives and potential targets for the diagnosis and treatment of OP.

\section{References}

1. Office of the Surgeon General (US): Bone Health and Osteoporosis: A Report of the Surgeon General. Office of the Surgeon General, Rockville, MD, pp379-382, 2004.

2. Beil FT, Seitz S, Priemel M, Barvencik F, von Domarus C, Rueger JM, Amling M and Pogoda P: Pathophysiology and pathomorphology of osteoporosis. Eur J Trauma Emerg Surg 34: $527-534,2008$.
3. Jimi E, Aoki K, Saito H, D'Acquisto F, May MJ, Nakamura I, Sudo T, Kojima T, Okamoto F, Fukushima H, et al: Selective inhibition of NF- $\kappa \mathrm{B}$ blocks osteoclastogenesis and prevents inflammatory bone destruction in vivo. Nat Med 10: 617-624, 2004.

4. Yamaguchi M and Weitzmann MN: The estrogen $17 \beta$-estradiol and phytoestrogen genistein mediate differential effects on osteoblastic NF-kB activity. Int J Mol Med 23: 297-301, 2009.

5. Vaira S, Johnson T, Hirbe AC, Alhawagri M, Anwisye I, Sammut B, O'Neal J, Zou W, Weilbaecher KN, Faccio R, et al: RelB is the NF- $\kappa \mathrm{B}$ subunit downstream of NIK responsible for osteoclast differentiation. Proc Natl Acad Sci USA 105: 3897-3902, 2008

6. Takano T, Li YJ, Kukita A, Yamaza T, Ayukawa Y, Moriyama K, Uehara N, Nomiyama H, Koyano K and Kukita T: Mesenchymal stem cells markedly suppress inflammatory bone destruction in rats with adjuvant-induced arthritis. Lab Invest 94: 286-296, 2014.

7. Robey PG and Termine JD: Human bone cells in vitro. Calcif Tissue Int 37: 453-460, 1985.

8. MacDonald BR, Takahashi N, McManus LM, Holahan J, Mundy GR and Roodman GD: Formation of multinucleated cells that respond to osteotropic hormones in long term human bone marrow cultures. Endocrinology 120: 2326-2333, 1987.

9. Svedbom A, Hernlund E, Ivergård M, Compston J, Cooper C Stenmark J, McCloskey EV, Jönsson B and Kanis JA; EU Review Panel of IOF: Osteoporosis in the European Union: A compendium of country-specific reports. Arch Osteoporos 8: 137, 2013

10. Dahlin DC: Bone remodeling dynamics. Am J Clin Pathol 1: 315-334, 1964

11. Morinaga T, Nakagawa N, Yasuda H, Tsuda E and Higashio K: Cloning and characterization of the gene encoding human osteoprotegerin/osteoclastogenesis-inhibitory factor. Eur J Biochem 254: 685-691, 1998.

12. Raisz LG: Pathogenesis of osteoporosis: Concepts, conflicts, and prospects. J Clin Invest 115: 3318-3325, 2005.

13. Qu Q, Perälä-Heape M, Kapanen A, Dahllund J, Salo J, Väänänen HK and Härkönen P: Estrogen enhances differentiation of osteoblasts in mouse bone marrow culture. Bone 22: 201-209, 1998.

14. Oreffo ROC, Kusec V, Virdi AS, Flanagan AM, Grano M, Zambonin-Zallone A and Triffitt JT: Expression of estrogen receptor-alpha in cells of the osteoclastic lineage. Histochem Cell Biol 111: 125-133, 1999.

15. Shevde NK, Bendixen AC, Dienger KM and Pike JW: Estrogens suppress RANK ligand-induced osteoclast differentiation via a stromal cell independent mechanism involving c-Jun repression. Proc Natl Acad Sci USA 97: 7829-7834, 2000.

16. Miller PD: Effects of parathyroid hormone and alendronate alone or in combination in osteoporosis. N Engl J Med 350: 189-192, 2004.

17. Obermayer-Pietsch B, Chararas C, Kotschan S, Walter D and Leb G: Genetic background of osteoporosis. Acta Med Austriaca 27: 18-22, 2000.

18. Xing L, Bushnell TP, Carlson L, Tai Z, Tondravi M, Siebenlist U, Young F and Boyce BF: NF- $\kappa$ B p50 and p52 expression is not required for RANK-expressing osteoclast progenitor formation but is essential for RANK- and cytokine-mediated osteoclastogenesis. J Bone Miner Res 17: 1200-1210, 2002.

19. Rosen CJ and Kessenich CR: The pathophysiology and treatment of postmenopausal osteoporosis. An evidence-based approach to estrogen replacement therapy. Endocrinol Metab Clin North Am 26: 295-311, 1997.

20. Miyazaki T, Katagiri H, Kanegae Y, Takayanagi H, Sawada Y, Yamamoto A, Pando MP, Asano T, Verma IM, Oda H, et al: Reciprocal role of ERK and NF- $\mathrm{BB}$ pathways in survival and activation of osteoclasts. J Cell Biol 148: 333-342, 2000.

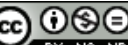

This work is licensed under a Creative Commons Attribution-NonCommercial-NoDerivatives 4.0 International (CC BY-NC-ND 4.0) License. 